\title{
Relativistic Ultrafast Electron Diffraction of Nanomaterials
}

Daniel Durham ${ }^{1}$, Khalid Siddiqui ${ }^{2}$, Fuhao $\mathrm{Ji}^{3}$, Jorge Giner Navarro ${ }^{4}$, Pietro Musumeci ${ }^{4}$, Robert Kaindl ${ }^{2}$, Andrew Minor ${ }^{1,2}$ and Daniele Filippetto ${ }^{2}$

${ }^{1}$ University of California, Berkeley, Berkeley, California, United States, ${ }^{2}$ Lawrence Berkeley National Laboratory, Berkeley, California, United States, ${ }^{3}$ SLAC National Accelerator Laboratory, Menlo Park, California, United States, ${ }^{4}$ University of California, Los Angeles, Los Angeles, California, United States

Dynamical ultrafast studies are increasingly becoming relevant for distinguishing nanoscale heterogeneity and probing nanoscale features, whether by full-field imaging or scanning nanoprobes. Relativistic ultrafast electron diffraction (UED) is well-suited for structural dynamics studies, allowing transmission through samples tens to hundreds of nanometers thick, large q-range, and many-electron bunches with femtosecond duration. However, MeV-scale UED probes are typically 10 s of microns wide, making application to ultrafast nanoscience challenging. At the High Repetition-rate Electron Scattering (HiRES) instrument at Lawrence Berkeley National Laboratory, we are bringing relativistic ultrafast electron diffraction to the nanoscale to enable a diverse range of nanoscience experiments.

HiRES utilizes the APEX gun, a high repetition-rate continuous-wave radiofrequency gun initially developed for x-ray free electron lasers [1]. This gun can deliver $750 \mathrm{keV}$ electron bunches at $\mathrm{MHz}$ repetition rates with bunch charge tunable from few to millions of electrons and duration tunable to less than 1 ps. High flux is itself a major advantage for nanomaterials studies where total scattering crosssections are minimal, such as in 2D materials and nanoscale objects. Furthermore, it affords significant collimation and manipulation of the beam to achieve smaller, shorter probes.

Taking advantage of APEX gun, we developed nanofocusing capabilities for the relativistic UED probes at HiRES with permanent magnet lenses [2]. With appropriate collimation, we achieved bunches with sub-nm normalized transverse emittance, allowing for simultaneous sub- $\mu \mathrm{m}$ spot size and sub-nm ${ }^{-1} \mathrm{k}-$ space resolution. With this, we were able to show scanning nanodiffraction across material domains and interfaces as well as point-projection imaging with sub- $\mu \mathrm{m}$ resolution. To characterize the beam itself, we imaged the probe in the far field while scanning a strongly scattering gold knife edge across the beam at various longitudinal positions, allowing us to reconstruct the full four-dimensional phase space of the electron beam [3]. A couple reconstructed beams are depicted in Figure 1, showing as small as sub-100 $\mathrm{nm}$ size in one dimension [2].

Recently, we have employed the HiRES instrument for pump-probe UED experiments on nanomaterials with a range of sample geometries and corresponding experimental requirements. Initial experiments have focused on ultrathin metals without nanofocusing. For instance, we studied the thermalization of excited electron-hole pairs in nanocrystalline cobalt thin films, observing 1.5 ps decay constants when pumping with 1030-nm wavelength laser pulses with $9 \mathrm{~mJ} \mathrm{~cm}^{-2}$ fluence (Figure 2a). While this experiment was performed at $500 \mathrm{~Hz}$ repetition rate, we can access higher repetition rates for future nanomaterials studies. For example, we have performed pump-probe UED on ultrathin $(11 \mathrm{~nm})$ single crystal gold films at varying repetition rates. Though the temporal response is similar at all repetition rates, change is observed at negative delay times when using higher repetition rates, corresponding to cumulative laser-induced heating. Still, the films can withstand up to several $\mathrm{kHz}$ pumping at $4 \mathrm{~mJ} \mathrm{~cm}^{-2}$ with a $650 \mu \mathrm{m}$ FWHM laser beam (Figure 2b). In addition, nanofocusing could allow pumping with smaller laser beams which 
may further reduce residual heating. These capabilities are now being applied to nanoscale and quantum materials experiments, such as ongoing studies of photoinduced phase transformations of $\mathrm{VO}_{2}$ nanowires (sample shown in Figure 2c).

a)

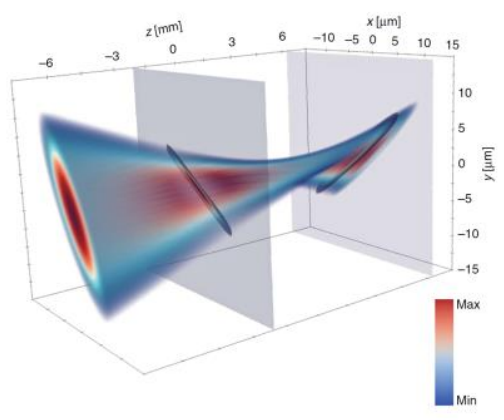

b)

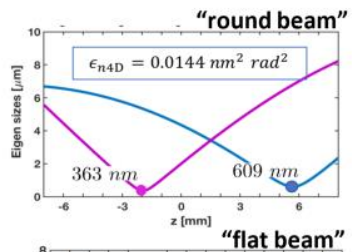

c)

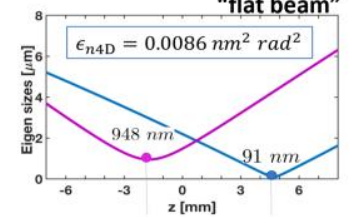

Figure 1. Ultrafast relativistic electron nanoprobes. (a) 3D reconstruction of an electron probe at HiRES around the beam waist after focusing with permanent magnet quadrupole lenses. (b) Corresponding RMS sizes of the beam over its propagation. (c) RMS sizes over propagation of a different magnetic lens configuration providing sub-100 nm width in one dimension. $\varepsilon n 4 \mathrm{D}$ is the normalized 4D emittance.

a) Nanocrystalline Co thin films

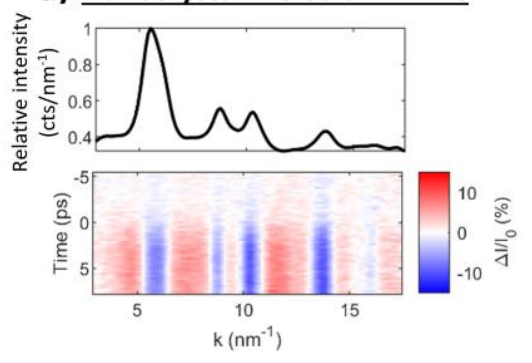

b) Ultrathin single-crystal gold

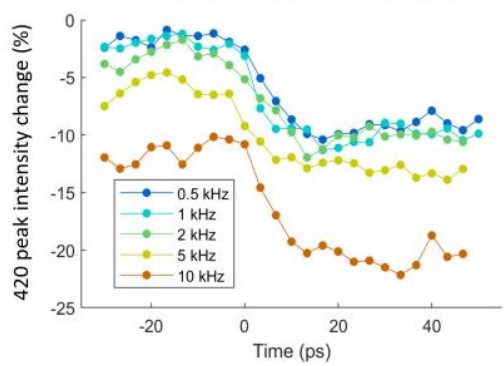

c) $\underline{\mathrm{VO}}_{2}$ nanowires

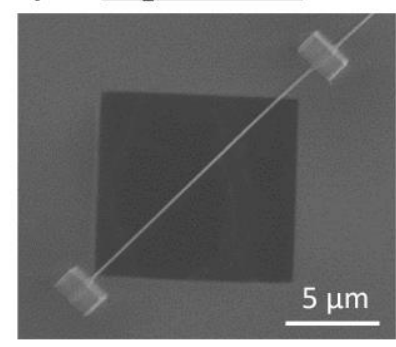

Figure 2. UED experiments at HiRES. (a) Electron-hole pair decay dynamics in nanocrystalline Cobalt thin films. Top panel is the initial azimuthally-integrated UED pattern, bottom is the time-dependent photoinduced change in the UED pattern, corresponding to lattice temperature rise. (b) UED of 11-nm thick freestanding gold films with 1030-nm wavelength laser pulses at $4 \mathrm{~mJ} \mathrm{~cm}-2$ fluence using varying repetition rate. Peak intensity change is between UED patterns acquired with pump laser on and those with pump laser off at a given pump-probe delay. (c) Scanning electron micrograph of a single-crystal VO2 nanowire sample prepared on a SiN TEM window for ongoing UED experiments.

\section{References}

[1] D. Filippetto and H. Qian, "Design of a high-flux instrument for ultrafast electron diffraction and microscopy," J. Phys. B: At., Mol. Opt. Phys, vol. 49, 104003, 2016.

[2] F. Ji, D. B. Durham, A. M. Minor, P. Musumeci, J. G. Navarro, and D. Filippetto, "Ultrafast relativistic electron nanoprobes," Communications Physics, vol. 2, no. 1, p. 54, 2019.

[3] F. Ji, J. G. Navarro, P. Musumeci, D. B. Durham, A. M. Minor, and D. Filippetto, "Knife-edge based measurement of the 4D transverse phase space of electron beams with picometer-scale emittance," Phys. Rev. Accel. Beams, vol. 22, no. 8, $082801,2019$. 